\title{
Circunferencia de la cintura como predictor de factores de riesgo de enfermedad cardiovascular en residentes costarricenses de 60 años y más
}

\author{
Eduardo Aguilar Fernández ${ }^{(1 D)}$ \& Ana Maricela Carballo Alfaro ${ }^{2}(D)$ \\ 1. Universidad Nacional, Facultad de Ciencias Exactas y Naturales, Escuela de Matemática, Heredia, Costa Rica; \\ eduardo.aguilar.fernandez@una.cr \\ 2. Hospital Nacional de Geriatría y Gerontología, Departamento de Farmacia, San José, Costa Rica; \\ anamaricelac@hotmail.com
}

Recibido 29-I-2021 • Corregido 04-III-2021 • Aceptado 22-III-2021

DOI: https://doi.org/10.22458/urj.v13i1.3398

\begin{abstract}
Waist circumference as a predictor of cardiovascular disease risk factors in Costa Rican residents aged 60 years and over". Introduction: There is a negative effect of abdominal obesity on the health of older adults. Objective: To investigate the relationship between waist circumference (WC) and metabolic risk factors associated with cardiovascular disease in this population. Methods: A population cross-sectional study with a total of 2418 people of the project Costa Rica: Longevity and Healthy Aging Study and referred to as CRELES. Spearman's correlation coefficient was calculated to estimate the correlations between WC with different risk factors for cardiovascular disease. Logistic regression analyses to calculate the odds ratio of a person presenting a cardiovascular risk factor according to the different WC levels. Results: People with wider waists are at greater risk of low HDL cholesterol, high triglycerides, diabetes and hypertension. In men, the strongest correlation was with triglycerides $(r=0,345)$, and in women with glycosylated hemoglobin $(r=0,262)$. Conclusions: Waist size can predict some risk factors for cardiovascular disease in Costa Rican residents aged 60 years and over.
\end{abstract}

Keywords: Obesity, risk factor, cardiovascular disease, anthropometry.
RESUMEN. Introducción: Existe un efecto negativo de la obesidad abdominal sobre la salud de los adultos mayores. Objetivo: Investigar la relación entre la circunferencia de cintura (CC) y los factores de riesgo metabólico asociados a la enfermedad cardiovascular en esta población. Métodos: El estudio incluye 2418 personas participantes en el proyecto Costa Rica: Estudio de Longevidad y Envejecimiento saludable (CRELES). Se calcularon coeficientes de correlación entre la CC y distintos factores de riesgo de enfermedad cardiovascular y modelos de regresión logística para calcular la razón de posibilidades que una persona presente un factor de riesgo cardiovascular según los distintos niveles de la CC. Resultados: Las personas con cinturas más anchas tienen un mayor riesgo de tener bajo colesterol HDL, altos niveles de triglicéridos, diabetes e hipertensión. En los hombres, la correlación más fuerte fue con los triglicéridos $(r=$ $0,345)$ y en las mujeres con hemoglobina glicosilada $(r=$ 0,262). Conclusiones: El tamaño de la cintura puede predecir algunos factores de riesgo de enfermedad cardiovascular en residentes de Costa Rica de 60 años y más.

Palabras clave: Obesidad, factor de riesgo, enfermedad cardiovascular, antropometría.

La obesidad es un importante problema de salud pública en el mundo (Afshin et al., 2017) y medidas como la circunferencia de la cintura (CC) han sido propuestas para evaluar el índice de obesidad, pues se ha considerado un buen indicador antropométrico de la obesidad abdominal (Ma et al., 2013). La CC considerada uno de los componentes del síndrome metabólico (National Cholesterol Education Program (NCEP) Expert Panel on Detection, Evaluation and Treatment of High Blood Cholesterol in Adults (Adult Treatment Panel III), 2002; Intenational Diabetes Federation, 2006) ha sido asociada con riesgo de desarrollar diabetes (Warren, Wilcox, Dowda, \& Baruth, 2012; Xin et al., 2012), hipertensión (Levine et al., 2011; Zhao et al., 2017), dislipidemia (Feng et al., 2012), enfermedad cardiovascular (Kazlauskienè, Butnorienè, \& Norkus, 2015) y mortalidad (Hotchkiss \& 
Leyland, 2011; Rost et al., 2018; Seidell, 2010; Staiano et al., 2012). Además, se ha mencionado que las personas con elevada CC tienen mayor probabilidad de desarrollar síndrome metabólico (Bener et al., 2013; Kim, Kim, \& Shin, 2020), o incluso, personas con un índice de masa corporal (IMC) normal pero con obesidad abdominal tienen mayor riesgo de sufrir hipertensión (Ostchega, Hughes, Terry, Fakhouri, \& Miller, 2012).

Por otro lado, tres niveles de la CC han sido determinados con el fin de evaluar el riesgo para la salud de las personas: riesgo normal (hombres: $\mathrm{CC}<94 \mathrm{~cm}$, mujeres: $\mathrm{CC}<80 \mathrm{~cm}$ ), riesgo para la salud en aumento o nivel de acción 1 (hombres: $94 \leq \mathrm{CC}<102 \mathrm{~cm}$, mujeres: $80 \leq C C<88 \mathrm{~cm}$ ) y riesgo para la salud alto o nivel de acción 2 (hombres: $C C \geq 102 \mathrm{~cm}$, mujeres: $\mathrm{CC} \geq 88 \mathrm{~cm}$ ) (Lean, Han, \& Morrison, 1995).

En el contexto de Costa Rica, se determinó que existe un efecto negativo de la obesidad abdominal en la salud de las personas adultas mayores hasta los 75 años de edad; sin embargo, para las personas con edades superiores los datos muestran la relación inversa, convirtiendo al sobrepeso en un factor protector, aunque en el caso de la obesidad abdominal el efecto protector es menor que el de la masa corporal (Rosero, Brenes, \& Méndez, 2009). En esta misma línea, se ha identificado que, en las personas mayores de 80 años, un IMC o una CC más grandes reducen el riesgo de morir (Rosero-Bixby \& Dow, 2012). Por otro lado, se ha determinado que existe relación entre los niveles actuales de los biomarcadores de riesgo cardiovascular con el índice de masa corporal actual, así como con el IMC máximo en el transcurso de la vida (Rehkopf, Duong, Dow, \& Rosero-Bixby, 2019).

El objetivo del presente estudio consistió en investigar el comportamiento de distintos factores de riesgo cardiovascular derivados de los biomarcadores en personas con 60 años o más, residentes en Costa Rica y categorizadas por niveles de riesgo para la salud previamente definidos para la circunferencia de la cintura.

\section{MATERIALES Y MÉTODOS}

Población de estudio: Para la presente investigación se consideró una muestra de 2418 personas participantes en la primera ronda de entrevistas del proyecto Costa Rica: Estudio de longevidad y envejecimiento saludable (CRELES), el cual es un estudio de tipo longitudinal basado en una muestra representativa de personas adultas mayores en Costa Rica, que residen en el país, sin tener en cuenta su nacionalidad, y cuyo nacimiento se haya presentado antes de 1946, es decir, tener 60 años o más al momento de la primera entrevista. En este proyecto se realizaron alrededor de 3000 entrevistas y la primera ronda se llevó a cabo entre noviembre de 2004 y setiembre de 2006. El proyecto fue desarrollado por el Centro Centroamericano de Población (CCP) y el Instituto de Investigaciones en Salud (INISA) de la Universidad de Costa Rica en colaboración con otras instituciones como la Caja Costarricense del Seguro Social (CCSS) y el Consejo Nacional de la Persona Adulta Mayor (CONAPAM) con fondos de la Welcome Trust Foundation. El estudio fue aprobado por el Comité de Ética Científica de la Universidad de Costa Rica en la sesión del 17 de marzo de 2004 (referencia: VI-763-CEC-23-04), proyecto de investigación número 828-A2 -825 (Rosero, Dow \& Fernández, 2013). En la presente investigación se consideraron todas personas participantes en la primera ronda de entrevistas que presentan información completa en todas las variables consideradas en el estudio.

\section{Variables de estudio}

Variables sociodemográficas: En este apartado se tomaron en cuenta la edad (variable continua), sexo (hombre o mujer), zona de residencia (urbana o rural) y educación (ninguna, primaria: 1 a 6 años de escolaridad, secundaria: 7 a 11 años y superior: 12 años o más). 
Prácticas de salud personal: Tabaquismo (si o no) ante la pregunta “¿Fuma usted actualmente?", consumo de bebidas alcohólicas (si consume o no bebidas alcohólicas de manera regular u ocasional) y actividad física (en dos categorías: si o no). Es importante aclarar en este punto que la actividad física hace referencia a "actividades y movimientos que incluyen actividades cotidianas, tales como caminar en forma regular, las tareas domésticas pesadas, bailar, y la jardinería entre otras" (Fernández \& Méndez, 2007, p. 6), mientras que el ejercicio físico es un tipo de actividad física que incluye "movimientos corporales planificados, estructurados y repetitivos, con el propósito de mejorar o mantener uno o más aspectos de la salud" (Fernández \& Méndez, 2007 , p. 6). Tomando en cuenta estas definiciones, en la presente investigación se consideró para la actividad física el tipo y frecuencia consultadas en CRELES a través de la pregunta: "En los últimos 12 meses, ¿̇hizo ejercicio con regularidad o realizó otras actividades físicas rigurosas como deportes, trotar, bailar o realizar trabajos pesados, tres veces por semana?"

Presión arterial (en mmHg: milímetros de mercurio): Se midió dos veces durante la entrevista principal de CRELES, con un intervalo de tiempo promedio de 20 minutos entre cada una.

Circunferencia de la cintura (en $\mathrm{cm}$ ): Las medidas abdominales en CRELES se realizaron con las personas participantes de pie, en posición semi-anatómica, es decir, con los pies separados y la palma de las manos apoyada en el lateral del muslo y para ello se utilizaron cintas métricas Dry y Quick Medical (Rosero-Bixby et al., 2013). Con el fin estudiar la relación de la CC con los factores de riesgo cardiovascular, se consideraron las tres categorías utilizadas para la evaluación del riesgo para la salud de las personas definidas anteriormente: $\mathrm{CC}<94 \mathrm{~cm}, 94 \leq \mathrm{CC}<102$ y CC $\geq 102$, en hombres y $C C<80,80 \leq C C<88$ y CC $\geq 88$, en mujeres (Lean et al., 1995).

Dieta: La información sobre la dieta de las personas participantes en CRELES fue obtenida a través de una versión abreviada del cuestionario de frecuencia de alimentos (FFQ por sus siglas en inglés), el cual fue desarrollado y validado específicamente para evaluar la ingesta de nutrientes en la población adulta de Costa Rica en el estudio de enfermedades coronarias (Rosero-Bixby et al., 2013). En la presente investigación se consideró las variables dieta alta en calorías (> $3000 \mathrm{kcal}$ por día), dieta alta en carbohidratos (> 400 g por día) y dieta alta en grasas saturadas ( 40 g por día), pues como lo mencionan Rehkopf, Dow y Rosero-Bixby (2010), estos valores son considerados puntos de corte estándar asociados con el riesgo de enfermedad cardiovascular.

Biomarcadores: Se consideraron el colesterol total (CT), el colesterol HDL (C-HDL), triglicéridos (TG), la hemoglobina glicosilada (HbA1c), la glucosa en ayunas (GL) y la proteína $C$ reactiva (PCR). Para la medición de los biomarcadores, en CRELES, se obtuvieron muestras de sangre mediante venopunción, normalmente durante la segunda visita, al día siguiente de la entrevista principal, con el participante en ayunas (durante 14 horas). Se recolectaron tres tubos de muestras de sangre: uno con anticoagulante (VACUTAINER / EDTA) de 3-4 ml que se centrifugó posteriormente para separar el plasma de las células y dos tubos sin anticoagulante con activador del coágulo (VACUTAINER SST, $5 \mathrm{ml}$ ) para la obtención de suero. En el laboratorio se separó una fracción de suero en un tubo cónico tipo Eppendorf para pruebas de CT, C-HDL, TG y GL y $1 \mathrm{ml}$ de sangre completa en el tubo EDTA para el análisis de HbA1c. Para el análisis de la PCR se utilizaron dos laboratorios, el del Centro Centroamericano de Análisis Hormonal y en el laboratorio del Hospital San Juan de Dios (HSJD). En el primero se utilizó el método "PCR de alta sensibilidad" con el equipo automatizado KONELAB TM y en el segundo utilizaron la agregación de partículas recubiertas con anticuerpos monoclonales anti-PCR y el equipo automatizado Dade Bohering BN System. Dado que los biomarcadores medidos a partir de muestras de sangre del Proyecto CRELES fueron analizados en diferentes laboratorios, se estableció el del HSJD como estándar y los resultados de los demás se ajustaron con ecuaciones estimadas por regresión en los grupos de validación (Rosero et al., 2013). En relación con los factores de riesgo cardiovascular, para la presente investigación se definieron como sigue: hipertensión si la persona presentó las siguientes 
características: medidas altas (PS $\geq 140 \mathrm{mmHg}$ o PD $\geq 90 \mathrm{mmHg}$, Chobanian et al., 2003) en 3 o más de las 4 mediciones realizadas durante la entrevista, se encontraba tomando medicamentos antihipertensivos o personal médico le ha dicho que es persona hipertensa. Colesterol alto si $C T \geq$ $250 \mathrm{mg} / \mathrm{dl}$, C-HDL bajo si C-HDL $\leq 40 \mathrm{mg} / \mathrm{dl}$ en hombres y C-HDL $\leq 50 \mathrm{mg} / \mathrm{dl}$ en mujeres y triglicéridos altos si TG $\geq 150 \mathrm{mg} / \mathrm{dl}$ (National Cholesterol Education Program (NCEP) Expert Panel on Detection, Evaluation and Treatment of High Blood Cholesterol in Adults (Adult Treatment Panel III), 2002). Diabetes mellitus Tipo 2 (diabetes) fue definida si la persona presenta alguna de las siguientes características: $\mathrm{HbA1c} \geq 6,5 \%$ (American Diabetes Association, 2018), se encontraba tomando medicamentos antidiabéticos o personal médico le ha dicho que es persona diabética. Elevada PCR se consideró si PCR $>3 \mathrm{mg} / \mathrm{l}$ en la prueba de alta sensibilidad, ya que estos valores son indicadores de alto riesgo de desarrollo de enfermedad cardiovascular (Ridker, 2016).

Análisis de los datos: Para el caso de las variables continuas, los resultados se presentaron como promedio y desviación estándar (DE) y como cantidad y porcentaje para variables categóricas. Se utilizó la prueba chi cuadrado para el análisis de las variables categóricas y la prueba t para la comparación de las variables continuas. Estos análisis toman en cuenta las ponderaciones muestrales.

Se utilizó el coeficiente de correlación de Spearman para estimar las correlaciones bivariadas entre la CC y los biomarcadores. También se calcularon correlaciones parciales ajustando por edad, zona de residencia, educación, tabaquismo, consumo de bebidas alcohólicas, actividad física, dieta alta en calorías, dieta alta en carbohidratos y dieta alta en grasas saturadas. Se estimaron modelos de regresión logística multivariada para calcular la razón de posibilidades (OR) de que una persona presente un factor de riesgo cardiovascular según los distintos niveles de la CC y por grupos de edad. Además, a partir de la estimación de los OR se determinó el tamaño del efecto en las asociaciones por grupo de edad ( $60-75$ y 76 o más) con el fin de identificar cambios en la magnitud del efecto de las relaciones cuando se comparan por edad tomando en consideración que la magnitud del efecto se considera insignificante si $O R<1,68$, pequeña si $1,68 \leq O R<3,47$, moderada si 3,47 $\leq O R<6,71$ y grande si $O R \geq 6,71$ (Chen, Cohen, $\&$ Chen, 2010). En el caso de OR $<1$, se utilizó el recíproco de los puntos de corte. Finalmente, los modelos multivariados fueron ajustados por edad, zona de residencia, educación, tabaquismo, consumo de bebidas alcohólicas, actividad física, dieta alta en calorías, dieta alta en carbohidratos y dieta alta en grasas saturadas.

Para la realización de los análisis estadísticos se utilizó el programa STATA versión 13.1 (stat Corp. USA) y los valores $p<0,05$ se consideraron estadísticamente significativos.

\section{RESULTADOS}

La edad promedio de las personas participantes fue de 70,2 años ( $D E=7,9$; edad máxima de 109 años). Las comparaciones por sexo indican niveles más altos de los biomarcadores en las mujeres, aunque las diferencias en TG, PS y PD no son significativas. Por otro lado, no se observaron diferencias significativas en la CC por sexo $(p=0,691)$; sin embargo, existe una elevada proporción de mujeres con obesidad abdominal (66,6\% vs $21,4 \%$ ). Además, los hombres son más propensos a fumar $(16,4 \%$ vs $3,9 \%)$, consumir bebidas alcohólicas $(31,2 \%$ vs $8,7 \%)$ y realizar actividad física (41,0 \% vs $24,5 \%$ ), mientras que el consumo de medicamentos (antihipertensivos, antidiabéticos y para el control de los lípidos) se da en mayor proporción en las mujeres (49,9\% vs $37,4 \% ; 20,7 \%$ vs $14,4 \%$ y $24,8 \%$ vs $18,4 \%$, respectivamente). En cuanto a la alimentación, existe mayor proporción de hombres con una dieta alta en calorías ( $16,8 \%$ vs $8,8 \%$ ), alta en carbohidratos $(20,4 \%$ vs $11,4 \%)$ y alta en grasas saturadas (15,5\% vs $11,7 \%)$ (Tabla 1$)$. 
TABLA 1

Costa Rica. Características generales de la población residente en el país con 60 años y más.

\begin{tabular}{|c|c|c|c|c|}
\hline Característica & $\begin{array}{c}\text { Total } \\
(n=2418)\end{array}$ & $\begin{array}{c}\text { Hombres } \\
(n=1099)\end{array}$ & $\begin{array}{c}\text { Mujeres } \\
(n=1319)\end{array}$ & $p$ \\
\hline Edad, (DE) & $70,2(7,9)$ & $69,8(7,8)$ & $70,6(8,0)$ & 0,037 \\
\hline$C C,(D E)$ & $93,5(12,4)$ & $93,4(10,7)$ & $93,6(13,7)$ & 0,691 \\
\hline$C T,(D E)$ & $216,2(49,7)$ & $205,4(46,1)$ & $226,0(50,7)$ & $<0,001$ \\
\hline C-HDL, (DE) & $44,2(13,1)$ & $40,7(12,0)$ & $47,4(13,3)$ & $<0,001$ \\
\hline $\mathrm{TG},(\mathrm{DE})$ & $165,5(93,0)$ & $162,9(95,2)$ & $167,9(91,0)$ & $<0,318$ \\
\hline HbA1c, (DE) & $5,7(1,1)$ & $5,6(0,9)$ & $5,9(1,2)$ & $<0,001$ \\
\hline $\mathrm{GL},(\mathrm{DE})$ & $110,8(45,7)$ & $107,3(43,3)$ & $113,9(47,6)$ & 0,005 \\
\hline PS, (DE) & $144,1(22,9)$ & $143,4(22,6)$ & $144,6(23,2)$ & 0,279 \\
\hline$P D,(D E)$ & $83,7(12,1)$ & $83,4(12,2)$ & $84,0(12,0)$ & 0,330 \\
\hline$P C R,(D E)$ & $5,6(7,1)$ & $5,3(7,5)$ & $6,0(6,7)$ & 0,022 \\
\hline Urbana, \% & 63,4 & 58,4 & 67,8 & $<0,001$ \\
\hline Tabaquismo, $\%$ & 9,8 & 16,4 & 3,9 & $<0,001$ \\
\hline Alcohol, \% & 19,3 & 31,2 & 8,7 & $<0,001$ \\
\hline Actividad física, \% & 32,3 & 41,0 & 24,5 & $<0,001$ \\
\hline Antihipertensivos, \% & 44,0 & 37,4 & 49,9 & $<0,001$ \\
\hline Antidiabéticos, \% & 17,7 & 14,4 & 20,7 & 0,001 \\
\hline Lípidos, \% & 21,8 & 18,4 & 24,8 & 0,003 \\
\hline Educación, \% & & & & 0,206 \\
\hline Ninguna & 13,6 & 13,9 & 13,3 & \\
\hline Primaria & 64,9 & 62,3 & 67,2 & \\
\hline Secundaria & 12,5 & 13,8 & 11,3 & \\
\hline Universitaria & 9,0 & 10,0 & 8,1 & \\
\hline Dieta alta en calorías, $\%$ & 12,3 & 16,1 & 8,8 & $<0,001$ \\
\hline Dieta alta en carbohidratos, \% & 15,7 & 20,4 & 11,4 & $<0,001$ \\
\hline Dieta alta en grasa saturada, $\%$ & 13,5 & 15,5 & 11,7 & 0,031 \\
\hline Obesidad abdominal*, \% & 45,2 & 21,4 & 66,6 & $<0,001$ \\
\hline
\end{tabular}

${ }^{*} \mathrm{CC} \geq 102 \mathrm{~cm}$ en hombres, $\mathrm{CC} \geq 88 \mathrm{~cm}$ en mujeres

Fuente: CRELES.

Los valores medios del C-HDL disminuyen mientras que para TG, PS, PD, HbA1c y GL tienden a aumentar con la CC en los hombres y mujeres. Por otro lado, la tendencia de crecimiento no resultó significativa para CT y PCR en ambos sexos y para TG $(p=0,362)$ en el caso de las mujeres (Tabla 2).

En el caso de la correlación de la CC con C-HDL, TG, HbA1c, GL y PD se obtuvieron coeficientes (en valor absoluto) mayores a 0,10. En ambos sexos, la correlación entre CT con CC no resultó significativa. Por otro lado, coeficientes de correlación de la CC y los biomarcadores cuyo valor absoluto fue mayor a 0,10 permanecieron significativas (excepto para la PCR en las mujeres) en las correlaciones parciales, luego de ajustar por edad, zona de residencia, educación, tabaquismo, consumo de bebidas alcohólicas, actividad física, dieta alta en calorías, dieta alta en carbohidratos y dieta alta en grasas saturadas. Además, la correlación más fuerte de la CC se dio con TG $(r=0,345)$ en los hombres y con la HbA1c $(r=0,262)$ en las mujeres (Tabla 3). 
TABLA 2

Nivel medio del biomarcador según las distintas categorías de la CC.

\begin{tabular}{|c|c|c|c|c|c|c|c|c|c|}
\hline \multirow{2}{*}{$\mathrm{CC}$} & \multirow{2}{*}{$\mathrm{n}$} & \multicolumn{8}{|c|}{ Biomarcador } \\
\hline & & CT & C-HDL & TG & PS & PD & GL & HbA1c & PCR \\
\hline \multicolumn{10}{|c|}{ Hombres ( $n=1099$ ) } \\
\hline$<94$ & 609 & 205,1 & 42,4 & 149,5 & 141,4 & 81,8 & 101,9 & 5,4 & 5,0 \\
\hline $94-101$ & 279 & 209,9 & 39,6 & 179,9 & 145,6 & 84,3 & 111,6 & 5,8 & 5,6 \\
\hline$\geq 102$ & 211 & 200,4 & 38,1 & 175,0 & 145,8 & 86,3 & 115,1 & 5,8 & 5,7 \\
\hline$p$ (tendencia) & & 0,488 & $<0,001$ & 0,001 & 0,021 & $<0,001$ & $<0,001$ & $<0,001$ & 0,151 \\
\hline \multicolumn{10}{|c|}{ Mujeres ( $n=1319)$} \\
\hline$<80$ & 210 & 228,9 & 51,6 & 159,1 & 142,2 & 81,7 & 102,2 & 5,5 & 5,5 \\
\hline $80-87$ & 281 & 233,3 & 49,4 & 167,1 & 143,3 & 83,3 & 109,3 & 5,6 & 5,5 \\
\hline$\geq 88$ & 828 & 223,2 & 45,9 & 169,9 & 145,5 & 84,7 & 117,6 & 6,0 & 6,2 \\
\hline$p$ (tendencia) & & 0,056 & $<0,001$ & 0,393 & 0,070 & 0,009 & 0,001 & $<0,001$ & 0,099 \\
\hline
\end{tabular}

Fuente: CRELES

TABLA 3

Correlación entre la CC y diferentes factores de riesgo cardiovascular

\begin{tabular}{lcccc}
\hline \multirow{2}{*}{ Biomarcador } & \multicolumn{2}{c}{ Hombre } & \multicolumn{2}{c}{ Mujer } \\
& Spearman & Parcial & Spearman & Parcial \\
\hline CT & $0,010^{*}$ & $-0,022^{*}$ & $-0,044^{*}$ & $-0,057$ \\
C-HDL & $-0,242$ & $-0,215$ & $-0,148$ & $-0,117$ \\
TG & 0,345 & 0,224 & 0,110 & 0,091 \\
HbA1c & 0,200 & 0,211 & 0,262 & 0,205 \\
GL & 0,279 & 0,193 & 0,215 & 0,139 \\
PS & 0,102 & 0,097 & $0,043^{*}$ & 0,055 \\
PD & 0,183 & 0,146 & 0,104 & 0,057 \\
PCR & $0,056^{*}$ & $0,037^{*}$ & 0,169 & $0,051^{*}$ \\
\hline
\end{tabular}

${ }^{*} p>0,5$

Fuente: CRELES

Por otro lado, la prevalencia de los factores de riesgo cardiovascular como bajo C-HDL, hipertensión o diabetes aumenta significativamente conforme incrementa la medida de la CC. Asimismo, se observa un crecimiento en la prevalencia de hipertensión conforme aumenta la edad en ambos sexos ( $58,1 \%$ vs $67,1 \% ; p=0,006$ en hombres; $67,6 \%$ vs $75,4 \% ; p=0,005$, en mujeres) y una disminución para la prevalencia de C-HDL bajo $(p=0,008)$ y triglicéridos altos $(p=0,004)$ en hombres. Las diferencias observadas en la diabetes no resultaron significativas por grupo de edad ( $p=0,084$ en hombres y $p=0,107$ en mujeres). Finalmente, se identificó que la hipertensión es el factor de riesgo que presenta mayor prevalencia tanto en hombres $(60,1 \%)$ como en mujeres $(69,5 \%)$ de 60 años y más (Tabla 4). 
TABLA 4

Prevalencia de los diferentes factores de riesgo cardiovascular por nivel de la CC.

\begin{tabular}{|c|c|c|c|c|c|c|}
\hline \multirow{2}{*}{ CC } & \multicolumn{6}{|c|}{ Factor de riesgo } \\
\hline & CT alto & C-HDL bajo & TG alto & Hipertensión & Diabetes & PCR alta \\
\hline \multicolumn{7}{|c|}{ Hombres Total ( $n=1099)$} \\
\hline Total & 15,0 & 54,1 & 42,6 & 60,1 & 18,5 & 53,0 \\
\hline$<94$ & 16,3 & 48,7 & 34,5 & 52,7 & 12,6 & 50,6 \\
\hline $94-101$ & 16,4 & 57,9 & 52,9 & 61,6 & 22,0 & 52,0 \\
\hline$\geq 102$ & 10,0 & 62,5 & 49,8 & 76,5 & 28,7 & 60,3 \\
\hline$p$ (tendencia) & 0,079 & 0,003 & $<0,001$ & $<0,001$ & $<0,001$ & 0,069 \\
\hline \multicolumn{7}{|c|}{ Edad $60-75(n=574)$} \\
\hline Total & 15,4 & 56,1 & 44,8 & 58,1 & 19,5 & 49,9 \\
\hline$<94$ & 17,1 & 49,4 & 36,7 & 50,6 & 13,8 & 46,5 \\
\hline $94-101$ & 17,1 & 62,4 & 56,9 & 58,1 & 23,6 & 48,7 \\
\hline$\geq 102$ & 9,6 & 64,5 & 49,7 & 75,5 & 28,2 & 59,2 \\
\hline$p$ (tendencia) & 0,085 & 0,006 & 0,007 & $<0,001$ & 0,001 & 0,049 \\
\hline \multicolumn{7}{|c|}{ Edad 76 o más $(n=525)$} \\
\hline Total & 13,6 & 47,1 & 35,2 & 67,1 & 15,0 & 63,6 \\
\hline$<94$ & 13,9 & 46,5 & 27,5 & 59,7 & 8,7 & 63,8 \\
\hline $94-101$ & 14,4 & 44,0 & 40,5 & 72,7 & 17,2 & 62,1 \\
\hline$\geq 102$ & 11,7 & 54,0 & 50,2 & 80,8 & 30,9 & 65,3 \\
\hline$p$ (tendencia) & 0,698 & 0,402 & $<0,001$ & $<0,001$ & $<0,001$ & 0,920 \\
\hline \multicolumn{7}{|c|}{ Mujeres Total ( $n=1319)$} \\
\hline Total & 31,2 & 64,7 & 47,4 & 69,5 & 27,1 & 65,9 \\
\hline$<80$ & 36,1 & 51,6 & 36,9 & 58,4 & 9,3 & 53,1 \\
\hline $80-87$ & 34,0 & 62,2 & 51,0 & 62,9 & 18,8 & 54,8 \\
\hline$\geq 88$ & 29,3 & 68,2 & 48,5 & 73,8 & 33,1 & 71,8 \\
\hline$p$ (tendencia) & 0,084 & $<0,001$ & 0,072 & $<0,001$ & $<0,001$ & $<0,001$ \\
\hline \multicolumn{7}{|c|}{ Edad $60-75(n=686)$} \\
\hline Total & 32,5 & 65,3 & 47,4 & 67,6 & 28,2 & 65,6 \\
\hline$<80$ & 38,8 & 51,2 & 37,8 & 57,9 & 11,4 & 51,0 \\
\hline $80-87$ & 37,1 & 61,1 & 50,2 & 59,8 & 19,4 & 51,8 \\
\hline$\geq 88$ & 30,0 & 68,8 & 48,3 & 71,4 & 33,6 & 72,0 \\
\hline$p$ (tendencia) & 0,079 & 0,003 & 0,232 & 0,006 & $<0,001$ & $<0,001$ \\
\hline \multicolumn{7}{|c|}{ Edad 76 o más $(n=633)$} \\
\hline Total & 27,3 & 63,2 & 47,3 & 75,4 & 23,8 & 66,6 \\
\hline$<80$ & 31,0 & 52,2 & 35,3 & 59,2 & 5,3 & 57,3 \\
\hline $80-87$ & 25,9 & 65,0 & 53,0 & 71,1 & 17,5 & 62,9 \\
\hline$\geq 88$ & 26,7 & 65,9 & 49,0 & 81,8 & 31,8 & 70,8 \\
\hline$p$ (tendencia) & 0,505 & 0,029 & 0,064 & $<0,001$ & $<0,001$ & 0,009 \\
\hline
\end{tabular}

Fuente: CRELES 
TABLA 5

Odds ratios (IC:95\%) de presentar factor de riesgo cardiovascular según las diferentes categorías de la CC.

\begin{tabular}{|c|c|c|c|c|c|c|}
\hline \multirow{2}{*}{$\mathrm{CC}$} & \multicolumn{6}{|c|}{ Factor de riesgo } \\
\hline & Alto $\mathrm{CT}$ & Bajo C-HDL & Alto TG & Hipertensión & Diabetes & Alta PCR \\
\hline \multicolumn{7}{|c|}{ Hombres Total ( $n=1099)$} \\
\hline$<94$ & 1,00 & 1,00 & 1,00 & 1,00 & 1,00 & 1,00 \\
\hline $94-101$ & $\begin{array}{c}1,01 \\
(0,67-1,51)\end{array}$ & $\begin{array}{c}1,40 \\
(1,04-1,89)\end{array}$ & $\begin{array}{c}2,09 \\
(1,55-2,82)\end{array}$ & $\begin{array}{c}1,56 \\
(1,16-2,11)\end{array}$ & $\begin{array}{c}1,83 \\
(1,24-2,72)\end{array}$ & $\begin{array}{c}1,07 \\
(0,80-1,44)\end{array}$ \\
\hline$\geq 102$ & $\begin{array}{c}0,73 \\
(0,45-1,18)\end{array}$ & $\begin{array}{c}1,66 \\
(1,18-2,31)\end{array}$ & $\begin{array}{c}2,40 \\
(1,72-3,34)\end{array}$ & $\begin{array}{c}3,31 \\
(2,26-4,84)\end{array}$ & $\begin{array}{c}3,25 \\
(2,18-4,85)\end{array}$ & $\begin{array}{c}1,56 \\
(1,11-2,18)\end{array}$ \\
\hline \multicolumn{7}{|c|}{ Edad $60-75(n=574)$} \\
\hline$<94$ & 1,00 & 1,00 & 1,00 & 1,00 & 1,00 & 1,00 \\
\hline $94-101$ & $\begin{array}{c}0,99 \\
(0,57-1,73)\end{array}$ & $\begin{array}{c}1,92 \\
(1,25-2,95)\end{array}$ & $\begin{array}{c}2,18 \\
(1,44-3,30)\end{array}$ & $\begin{array}{c}1,43 \\
(0,94-2,16)\end{array}$ & $\begin{array}{c}1,97 \\
(1,17-3,32)\end{array}$ & $\begin{array}{c}1,11 \\
(0,80-1,43)\end{array}$ \\
\hline$\geq 102$ & $\begin{array}{c}0,64 \\
(0,33-1,23)\end{array}$ & $\begin{array}{c}1,99 \\
(1,27-3,14)\end{array}$ & $\begin{array}{c}2,05 \\
(1,33-3,18)\end{array}$ & $\begin{array}{c}3,84 \\
(2,32-6,36)\end{array}$ & $\begin{array}{c}3,14 \\
(1,87- \\
5,27)\end{array}$ & $\begin{array}{c}1,81 \\
(1,16-2,81)\end{array}$ \\
\hline \multicolumn{7}{|c|}{ Edad 76 o más $(n=525)$} \\
\hline$<94$ & 1,00 & 1,00 & 1,00 & 1,00 & 1,00 & 1,00 \\
\hline $94-101$ & $\begin{array}{c}1,02 \\
(0,55-1,88)\end{array}$ & $\begin{array}{c}1,07 \\
(0,70-1,65)\end{array}$ & $\begin{array}{c}1,92 \\
(1,23-3,01)\end{array}$ & $\begin{array}{c}1,62 \\
(1,03-2,55)\end{array}$ & $\begin{array}{c}1,79 \\
(0,96-3,33)\end{array}$ & $\begin{array}{c}1,15 \\
(0,74-1,81)\end{array}$ \\
\hline$\geq 102$ & $\begin{array}{c}0,84 \\
(0,40-1,76)\end{array}$ & $\begin{array}{c}1,36 \\
(0,81-2,26)\end{array}$ & $\begin{array}{c}2,80 \\
(1,66-4,72)\end{array}$ & $\begin{array}{c}2,49 \\
(1,38-4,47)\end{array}$ & $\begin{array}{c}3,76 \\
(1,96-7,19)\end{array}$ & $\begin{array}{c}1,54 \\
(0,77-2,24)\end{array}$ \\
\hline \multicolumn{7}{|c|}{ Mujeres Total $(n=1319)$} \\
\hline$<80$ & 1,00 & 1,00 & 1,00 & 1,00 & 1,00 & 1,00 \\
\hline $80-87$ & $\begin{array}{c}0,78 \\
(0,53-1,17)\end{array}$ & $\begin{array}{c}1,55 \\
(1,08-2,24)\end{array}$ & $\begin{array}{c}1,82 \\
(1,25-2,66)\end{array}$ & $\begin{array}{c}1,55 \\
(1,06-2,26)\end{array}$ & $\begin{array}{c}2,57 \\
(1,39-4,76)\end{array}$ & $\begin{array}{c}1,03 \\
(0,72-1,50)\end{array}$ \\
\hline$\geq 88$ & $\begin{array}{c}0,73 \\
(0,52-1,02)\end{array}$ & $\begin{array}{c}2,01 \\
(1,46-2,76)\end{array}$ & $\begin{array}{c}1,93 \\
(1,38-2,68)\end{array}$ & $\begin{array}{c}2,63 \\
(1,89-3,66)\end{array}$ & $\begin{array}{c}5,82 \\
(3,35- \\
10,11)\end{array}$ & $\begin{array}{c}1,76 \\
(1,28-2,43)\end{array}$ \\
\hline \multicolumn{7}{|c|}{ Edad $60-75(n=686)$} \\
\hline$<80$ & 1,00 & 1,00 & 1,00 & 1,00 & 1,00 & 1,00 \\
\hline $80-87$ & $\begin{array}{c}0,91 \\
(0,50-1,66)\end{array}$ & $\begin{array}{c}1,44 \\
(0,81-2,57)\end{array}$ & $\begin{array}{c}1,64 \\
(0,91-2,96)\end{array}$ & $\begin{array}{c}1,36 \\
(0,75-2,47)\end{array}$ & $\begin{array}{c}1,85 \\
(0,80-4,25)\end{array}$ & $\begin{array}{c}1,10 \\
(0,62-1,96)\end{array}$ \\
\hline$\geq 88$ & $\begin{array}{c}0,70 \\
(0,42-1,16)\end{array}$ & $\begin{array}{c}2,07 \\
(1,26-3,39)\end{array}$ & $\begin{array}{c}1,76 \\
(1,06-2,94)\end{array}$ & $\begin{array}{c}2,04 \\
(1,22-3,40)\end{array}$ & $\begin{array}{c}4,08 \\
(1,97-8,45)\end{array}$ & $\begin{array}{c}2,11 \\
(1,29-3,48)\end{array}$ \\
\hline \multicolumn{7}{|c|}{ Edad 76 o más $(n=633)$} \\
\hline$<80$ & 1,00 & 1,00 & 1,00 & 1,00 & 1,00 & 1,00 \\
\hline $80-87$ & $\begin{array}{c}0,63 \\
(0,36-1,11)\end{array}$ & $\begin{array}{c}1,54 \\
(0,95-2,51)\end{array}$ & $\begin{array}{c}1,87 \\
(1,13-3,11)\end{array}$ & $\begin{array}{c}1,58 \\
(0,96-2,64)\end{array}$ & $\begin{array}{c}3,52 \\
(1,37-9,02)\end{array}$ & $\begin{array}{c}0,98 \\
(0,61-1,60)\end{array}$ \\
\hline$\geq 88$ & $\begin{array}{c}0,76 \\
(0,47-1,22)\end{array}$ & $\begin{array}{c}1,94 \\
(1,27-2,96)\end{array}$ & $\begin{array}{c}2,01 \\
(1,28-3,15)\end{array}$ & $\begin{array}{c}3,02 \\
(1,91-4,80)\end{array}$ & $\begin{array}{c}7,83 \\
(3,31- \\
18,53) \\
\end{array}$ & $\begin{array}{c}1,46 \\
(0,95-2,24)\end{array}$ \\
\hline
\end{tabular}

Fuente: CRELES

Los OR de presentar C-HDL bajo, triglicéridos altos, hipertensión o diabetes en hombres en el nivel de acción 2 (CC $\geq 102 \mathrm{~cm}$ ) en comparación con aquellos con CC < $94 \mathrm{~cm}$ fueron 1,66 (IC95\%: 1,18 - 2,31), 2,40 (IC95\%: 1,72 - 3,34), 3,31 (IC95\%: 2,26 - 4,84) y 3,25 (2,18 - 4,85), respectivamente. En el caso de las mujeres en el nivel de acción $2(\mathrm{CC} \geq 88 \mathrm{~cm})$ en comparación con aquellas con CC < $80 \mathrm{~cm}$, los OR fueron 2,01 (IC95\%: 1,46 - 2,76), 1,93 (IC95\%: 1,38 - 2,68), 2,63 (IC95\%: $1,89-3,66)$ y $5,82(3,35-10,11)$, respectivamente. En el caso de la PCR, si bien no se obtuvo 
una correlación importante con la CC en hombres, los OR reflejan que las personas de ambos sexos con mediciones de CC en el nivel de acción 2 tienen mayor riesgo de presentar PCR $>3 \mathrm{mg} / \mathrm{l}(1,56$ para hombres y 1,76 para mujeres). Finalmente, los OR para el CT no resultaron significativos. Por otro lado, aunque los cambios en los OR por grupos de edad no resultaron significativos (valores $p$ superiores a 0,05), si se observó una disminución en el tamaño del efecto para bajo C-HDL, hipertensión y alta PCR (de pequeño a insignificante, de moderado a pequeño y de pequeño a insignificante, respectivamente) en los hombres con elevada CC para los mayores de 75 años, mientras que en las mujeres se dio una disminución en el OR para bajo C-HDL y alta PCR; sin embargo, solo en la PCR se presentó un cambio en la magnitud del efecto (de pequeño a insignificante). Además, se encontró que para las personas que llegan a edades avanzadas con CC en el nivel de acción 2, hay un aumento en la magnitud del efecto (de moderado a grande) para la diabetes si se compara con el grupo de 60 a 75 años (Tabla 5).

\section{DISCUSIÓN}

El estudio analizó las relaciones entre diversos factores de riesgo de enfermedad cardiovascular y la CC en personas de 60 años y más participantes en el proyecto CRELES. Los resultados mostraron que las correlaciones de la CC con C-HDL, triglicéridos, glucosa y HbA1c son significativas e independientes de la edad y otras variables confusoras como educación y tabaquismo.

Por otro lado, estudios previos han mencionado que la CC está fuertemente relacionada con los factores de riesgo de enfermedad cardiovascular (Van Dijk, Takken, Prinsen, \& Wittink, 2012). Esta afirmación se verifica con los resultados de la investigación, pues los modelos logísticos determinaron que conforme incrementa la medida de la CC, existe un mayor riesgo de presentar CHDL bajo, TG altos, hipertensión o diabetes aún cuando se incorporaran variables sociodemográficas y relacionadas con la salud.

Además, si se compara por sexo, se observa que en las mujeres con elevada CC existe mayor riesgo de desarrollar bajo C-HDL y diabetes, mientras que en los hombres existe mayor riesgo de presentar TG altos e hipertensión. Si se compara por grupos de edad, la probabilidad de desarrollar un factor de riesgo disminuye en algunos casos para las personas de mayor edad pero aumenta en otros. Un comportamiento particular se da para la diabetes, pues en mujeres de 76 años o más con elevada CC el riesgo de padecerla es de 7,83, es decir, 3,75 más si se compara con las del grupo de menor edad. Estudios previos han indicado que en las mujeres iraníes (Mohammadifard et al., 2013) como en mujeres afroamericanas (Warren et al., 2012), la CC es un fuerte predictor de diabetes. Esta afirmación se verifica en la investigación, pues las mujeres que presentaban una elevada CC (nivel de acción 2), el riesgo de desarrollar diabetes es hasta 4 veces o más si se compara con el riesgo de aquellas que presentaban una medida por debajo del nivel de acción 1 . En los hombres, este riesgo es superior a 3. Por otro lado, en el caso de la hipertensión el riesgo es 2 veces o más en ambos sexos y en los distintos grupos de edad. Estos resultados respaldan la afirmación de que la CC se considera un buen predictor de diabetes (The InterAct Consortium, 2012) y una herramienta clínica sencilla para evaluar el riesgo de hipertensión entre las personas con obesidad (Dimitriadis et al., 2016). Los hallazgos también revelaron que la probabilidad de padecer un determinado factor de riesgo cardiovascular no aumenta en todos los casos para las personas de mayor edad que presentan obesidad abdominal, esto quizás respaldado por el efecto protector que ejerce una CC más grande sobre la salud de las personas de edades avanzadas; sin embargo, es importante poner atención al efecto producido por la presencia de una CC elevada en la diabetes, en especial en las mujeres, dada la alta prevalencia de obesidad abdominal en ellas después de los 60 años (66,6\% para la población en estudio, 59,7\% para las mayores de 75 años) y además, porque existen análisis 
que indican la presencia de una relación positiva entre $\mathrm{HbA1c}$ y mortalidad en la población adulta mayor costarricense (Rosero-Bixby \& Dow, 2012).

Por otro lado, no se encontró relación entre CC y CT, mientras que para las personas con medida de la CC en el nivel de acción 2 existe riesgo de presentar PCR alta en las edades de 75 o menos. Un estudio previo también encontró relación entre CC y PCR en personas menores de 60 años (Aguilar \& Carballo, 2020). Aunque en las personas adultas mayores de más edad no se encontró relación significativa entre CC y PCR, es importante tomar en cuenta que la falta de relación entre la CC y un factor de riesgo de enfermedad cardiovascular podría ocurrir porque el poder predictivo de los indicadores antropométricos depende de la población de estudio (Molarius \& Seidell, 1998).

Entre las limitaciones de la investigación destaca la naturaleza de corte transversal de la muestra, ya que solo se consideraron los datos de la ronda 1 de CRELES, situación que provoca la imposibilidad de detectar cambios en el comportamiento de los factores de riesgo en el tiempo así como la de establecer relaciones causales. Además, los datos provienen de personas de 60 años y más, por lo que las conclusiones del estudio no pueden considerarse para quienes se encuentran fuera de ese rango. Finalmente, las mediciones de PCR analizadas son producto de la toma de una muestra de sangre lo cual no permite contar con estimaciones más estables para la PCR como las que proporcionarían el promedio de dos ensayos en condiciones óptimas con 2 semanas de diferencia (Pearson et al., 2003).

Entre las principales conclusiones pueden mencionarse que la investigación confirma la existencia de asociación entre la CC con distintos factores de riesgo de enfermedad cardiovascular, es decir, la presencia de una CC más grande es un predictor independiente de factores de riesgo cardiovascular. Además, aunque su efecto puede disminuir con la edad en algunos de los factores, su valor predictivo sigue siendo relevante, ya que mantiene su significancia estadística. Se ha mencionado que los niveles de acción identificados a partir de la medida de la CC pueden proporcionar un método valioso y simple para brindar información a las personas con mayor riesgo de enfermedad cardiovascular sobre el beneficio que podrían obtener de todas aquellas acciones orientadas al control del peso (Han, van Leer, Seidell, \& Lean, 1995). Además, la medición de la CC en la práctica clínica es fundamental para identificar a pacientes metabólicamente obesos y con sobrepeso cuyo IMC es normal y, por lo tanto, quedarían excluidos de intervenciones y tratamientos en el estilo de vida (Cetin, Lessig, \& Nasr, 2016).

Finalmente, estos resultados respaldan la idea de que la CC es un predictor de factores de riesgo de enfermedad cardiovascular en las personas de 60 años y más que residen en el territorio costarricense. Además, es importante motivar a las instancias encargadas de la salud pública a la continua elaboración y aplicación de programas de prevención y control de la obesidad así como de promoción de la salud que se orienten a controlar la aparición de factores de riesgo cardiovascular no solo para esta población sino en los grupos de edades más jóvenes, tomando en consideración que en el país la prevalencia de obesidad en personas mayores de 18 años pasó del 6,4\% en el año 1980 a 25,7\% en el 2016 (Organización de las Naciones Unidas para la Alimentación y la Agricultura, 2018) y que para el 2018 el 30,9\% de las personas adolescentes presentan sobrepeso u obesidad (Ministerio de Salud de Costa Rica, 2020), situación que obliga a realizar cambios en el patrón nutricional de la población. 


\section{AGRADECIMIENTOS}

Agradecemos al proyecto CRELES (Costa Rica: Estudio de Longevidad y Envejecimiento Saludable) es un estudio longitudinal de la Universidad de Costa Rica, realizado por el Centro Centroamericano de Población en colaboración con el Instituto de Investigaciones en Salud, con el apoyo de la Wellcome Trust Foundation. Investigador principal: Luis Rosero-Bixby. Coinvestigadores: Xinia Fernández y William H. Dow. Investigadores colaboradores: Ericka Méndez, Guido Pinto, Hannia Campos, Kenia Barrantes, Floribeth Fallas, Gilbert Brenes y Fernando Morales. Personal informático y de apoyo: Daniel Antich, Aaron Ramírez, Jeisson Hidalgo, Juanita Araya and Yamileth Hernández. Personal de campo: José Solano, Julio Palma, Jenny Méndez, Maritza Aráuz, Mabelyn Gómez, Marcela Rodríguez, Geovanni Salas, Jorge Vindas and Roberto Patiño.

\section{ÉTICA, CONFLICTO DE INTERESES Y DECLARACIÓN DE FINANCIAMIENTO}

Las personas autoras declaran haber cumplido con todos los requisitos éticos y legales pertinentes, tanto durante el estudio como en el manuscrito; que no hay conflictos de interés de ningún tipo, y que todas las fuentes financieras se detallan plena y claramente en la sección de agradecimientos. Asimismo, están de acuerdo con la versión editada final del documento. El respectivo documento legal firmado se encuentra en los archivos de la revista.

La contribución de los autores es como se detalla a continuación: E.A.F.: investigador, análisis de datos y redacción del artículo. A.M.C.A.: investigadora y redacción del artículo.

\section{REFERENCIAS}

Aguilar, E., \& Carballo, A. (2020). Factores asociados a la Proteína C en la población costarricense nacida entre 1945 y 1955. Población y Salud en Mesoamérica, 17(2). DOI: 10.15517/psm.v17i2.39798

American Diabetes Association (2018). 2. Classification and diagnosis of diabetes: standards of medical care in diabetes. Diabetes Care 42(1), 13-28. DOI: 10.2337/dc18-S002

Afshin, A., Forouzanfar, M. H., Reitsma, M. B., Sur, P., Estep, K., Lee, A., ... Murray, C. (2017). Health effects of overweight and obesity in 195 countries over 25 years. The New England journal of medicine, 377(1), 13-27. DOI: 10.1056/NEJMoa1614362

Bener, A., Yousafzai, M. T., Darwish, S., Al-Hamaq, A. O., Nasralla, E. A., \& Abdul-Ghani, M. (2013). Obesity index that better predict metabolic syndrome: body mass index, waist circumference, waist hip ratio, or waist height ratio. Journal of obesity, 2013. DOI: 10.1155/2013/269038

Cetin, D., Lessig, B. A., \& Nasr, E. (2016). Comprehensive evaluation for obesity: beyond body mass index. Journal of American Osteopathic Association, 116(6), 376-382. DOI: 10.7556/jaoa.2016.078

Chen, H., Cohen, P., \& Chen, S. (2010). How big is a big odds ratio? Interpreting the magnitudes of odds ratios in epidemiological studies. Communications in Statistics-Simulation and Computation, 39(4), 860-864. DOI: $10.1080 / 03610911003650383$

Chobanian, A.V., Bakris, G.L., Black, H.R., Cushman, W.C., Green, L.A., Izzo, J.L. Jr., . . the National High Blood Pressure Education Program Coordinating Committee (2003). The seventh report of the Joint National Committee on Prevention, Detection, Evaluation, and Treatment of High Blood Pressure: the JNC 7 Report. Hypertension, 42, 1206-1252. DOI: 10.1161/01.HYP.0000107251.49515.c2 
Dimitriadis, K., Tsioufis, C., Mazaraki, A., Liatakis, I., Koutra, E., Kordalis, A., ... \& Tousoulis, D. (2016). Waist circumference compared with other obesity parameters as determinants of coronary artery disease in essential hypertension: a 6-year follow-up study. Hypertension Research, 39(6), 475-479. DOI: 10.1038/hr.2016.8

Fernández, X., \& Méndez, E. (2007). Estilos de vida y factores de riesgo para la salud de las personas adultas mayores del proyecto CRELES-Costa Rica 2004-2006. Población y salud en Mesoamérica, 5(1), 1-17. DOI: 10.15517/PSM.V5I1.4547

Feng, R. N., Zhao, C., Wang, C., Niu, Y. C., Li, K., Guo, F. C., ... \& Li, Y. (2012). BMI is strongly associated with hypertension, and waist circumference is strongly associated with type 2 diabetes and dyslipidemia, in northern Chinese adults. Journal of epidemiology, 22(4), 317-323. DOI: 10.2188/jea.JE20110120

Han, T. S., van Leer, E. M., Seidell, J. C., \& Lean, M. E. (1995). Waist circumference action levels in the identification of cardiovascular risk factors: prevalence study in a random sample. BMJ (Clinical research ed.), 311(7017), 14011405. DOI: $10.1136 / \mathrm{bmj} .311 .7017 .1401$

Hotchkiss, J.W., \& Leyland, A.H. (2011). The relationship between body size and mortality in the linked Scottish Health Surveys: cross-sectional surveys with follow-up. International journal of obesity, 35(6), 838-851. DOI: 10.1038/ijo.2010.207.

International Diabetes Federation (2006). The IDF consensus worldwide definition of the metabolic syndrome. Recuperado de www.idf.org

Kazlauskienè, L., Butnorienè, J. \& Norkus, A. (2015). Metabolic syndrome related to cardiovascular events in a 10-year prospective study. Diabetology \& metabolic syndrome, 7(102), 1-7. DOI: 10.1186/s13098-015-0096-2

Kim, H., Kim, K., \& Shin, S. (2020). Cardiometabolic risk factor in obese and normal weight individuals in community dwelling men. International journal of environmental research and public health, 17, 8925. DOI: 10.3390/ijerph17238925

Lean, M. E., Han, T. S., \& Morrison, C. E. (1995). Waist circumference as a measure for indicating need for weight management. BMJ, 311(6998), 158-161. DOI: 10.1136/bmj.311.6998.158

Levine, D. A., Calhoun, D. A., Prineas, R. J., Cushman, M., Howard, V. J., \& Howard, G. (2011). Moderate waist circumference and hypertension prevalence: the REGARDS Study. American journal of hypertension, 24(4), 482-488. DOI: 10.1038/ajh.2010.258

Ma, W. Y., Yang, C. Y., Shih, S. R., Hsieh, H. J., Hung, C. S., Chiu, F. C., ... \& Li, H. Y. (2013). Measurement of waist circumference: midabdominal or iliac crest?. Diabetes care, 36(6), 1660-1666. DOI: doi.org/10.2337/dc12-145

Ministerio de Salud Pública de Costa Rica (2020). Informe de resultados encuesta colegial de vigilancia nutricional y actividad física. Recuperado de https://www.ministeriodesalud.go.cr/index.php/biblioteca-de-archivos/centrode-informacion/material-publicado/investigaciones/encuestas-de-salud/censo-escolar-peso-talla/encuestacolegial-de-vigilancia-nutricional-y-actividad-fisica-2018/4622-informe-de-resultados-encuesta-colegial-devigilancia-nutricional-y-actividad-fisica-2018/file

Mohammadifard, N., Nazem, M., Sarrafzadegan, N., Nouri, F., Sajjadi, F., Maghroun, M., \& Alikhasi, H. (2013). Body mass index, waist-circumference and cardiovascular disease risk factors in Iranian adults: Isfahan healthy heart program. Journal of health, population, and nutrition, 31(3), 388. DOI: 10.3329/jhpn.v31i3.16831

Molarius, A., \& Seidell, J. C. (1998). Selection of anthropometric indicators for classification of abdominal fatness--a critical review. International journal of obesity and related metabolic disorders, 22(8), 719-727. DOI: 10.1038/sj.ijo.0800660

National Cholesterol Education Program (NCEP) Expert Panel on Detection, Evaluation and Treatment of High Blood Cholesterol in Adults (Adult Treatment Panel III) (2002). Third Report of the National Cholesterol Education Program (NCEP) Expert Panel on detection, Evaluation, and Treatment of High Blood Cholesterol in Adults (Adult Treatment Panel III) Final Report. Circulation, 106(25), 3143-3421. DOI: 10.1161/circ.106.25.3143 
Organización de las Naciones Unidas para la Alimentación y la Agricultura (2018). Panorama de la seguridad alimentaria y nutricional en América Latina y el Caribe. Recuperado de http://www.fao.org/3/CA2127ES/CA2127ES.pdf

Ostchega, Y., Hughes, J. P., Terry, A., Fakhouri, T. H., \& Miller, I. (2012). Abdominal obesity, body mass index, and hypertension in US adults: NHANES 2007-2010. American journal of hypertension, 25(12), 1271-1278. DOI: 10.1038/ajh.2012.120

Rehkopf, D. H., Duong, A., Dow, W. H., \& Rosero-Bixby, L. (2019). Life-course BMI and biomarkers in persons aged 60 years or older: a comparison of the USA and Costa Rica. Public health nutrition, 22(2), 314-323. DOI: $10.1017 / \mathrm{S} 1368980018002276$

Rehkopf, D. H., Dow, W. H., \& Rosero-Bixby, L. (2010). Differences in the association of cardiovascular risk factors with education: a comparison of Costa Rica (CRELES) and the USA (NHANES). Journal of Epidemiology \& Community Health, 64(9), 821-828. DOI: 10.1136/jech.2009.086926

Ridker, P. M. (2016). A test in context: high-sensitivity C-reactive protein. Journal of the American College of Cardiology, 67(6), 712-723. DOI: 10.1016/j.jacc.2015.11.037

Rosero, L., Brenes G., \& Méndez, E. (2009) Obesidad, envejecimiento y mortalidad en Costa Rica. En E. Peláez (Ed.), Sociedad y adulto mayor en América Latina. Estudios sobre envejecimiento en la región (pp117-137). Brasil: ALAP. Recuperado de http://www.kerwa.ucr.ac.cr/handle/10669/29245

Rosero-Bixby, L., \& Dow, W.H. (2012). Predicting mortality with biomarkers: a population-based prospective cohort study for elderly Costa Ricans. Population health metrics 10(11), 1-15. DOI:10.1186/1478-7954-10-11

Rosero-Bixby, L., Dow, W.H., \& Fernández, X. (2013). CRELES: Costa Rican Longevity and Healthy Aging Study. Methods, Wave 1. Berkeley, CA: Department of Demography, University of California, Berkeley [Electronic Document]. Recuperado de http://www.creles.berkeley.edu/pdf/Methods_w1.pdf

Rost, S., Freuer, D., Peters, A., Thorand, B., Holle, R., Linseisen, J., \& Meisinger, C. (2018). New indexes of body fat distribution and sex-specific risk of total and cause-specific mortality: a prospective cohort study. BMC Public Health, 18(1), 427. DOI: 10.1186/s12889-018-5350-8.

Seidell, J. (2010). Waist circumference and waist/hip ratio in relation to all-cause mortality, cancer and sleep apnea. European journal of clinical nutrition, 64, 35-41. DOI: 10.1038/ejcn.2009.71

Staiano, A.E., Reeder, B.A., Elliott, S., Joffres, M.R., Pahwa, P., Kirkland, S.A., . . Katzmarzyk, P.T. (2012). Body mass index versus waist circumference as predictors of mortality in Canadian adults. International journal of obesity, 36(11), 1450-1454. DOI: 10.1038/ijo.2011.268

The InterAct Consortium (2012). Long-term risk of incident Type 2 Diabetes and measures of overall and regional obesity: The EPIC-InterAct Case-Cohort Study. PLOS Medicine 9(6): e1001230. DOI: 10.1371/journal.pmed.1001230

Van Dijk, S. B., Takken, T., Prinsen, E. C., \& Wittink, H. (2012). Different anthropometric adiposity measures and their association with cardiovascular disease risk factors: a meta-analysis. Netherlands heart journal, 20(5), 208-218. DOI: $10.1007 / \mathrm{s} 12471-011-0237-7$

Warren, T. Y., Wilcox, S., Dowda, M., \& Baruth, M. (2012). Independent association of waist circumference with hypertension and diabetes in African American women, South Carolina, 2007-2009. Preventing chronic disease, 9, E105. DOI: 10.5888/pcd9.110170

Xin, Z., Liu, C., Niu, W. Y., Feng, J. P., Zhao, L., Ma, Y. H., ... \& Yang, J. K. (2012). Identifying obesity indicators which best correlate with type 2 diabetes in a Chinese population. BMC Public Health, 12(1), 1-6. DOI: 10.1186/1471-2458$12-732$

Zhao, Y., Zhang, M., Luo, X., Wang, C., Li, L., Zhang, L., ... \& Hu, D. (2017). Association of 6-year waist circumference gain and incident hypertension. Heart, 103(17), 1347-1352. DOI: 10.1136/heartjnl-2016-310760 